\title{
Experimental investigation of bubble formation during capillary filling of $\mathrm{SiO2}$ nanoslits
}

Thamdrup, Lasse Højlund; Persson, Karl Fredrik; Bruus, Henrik; Kristensen, Anders; Flyvbjerg, Henrik

Published in:

Applied Physics Letters

Link to article, DOI:

$10.1063 / 1.2801397$

Publication date:

2007

Document Version

Publisher's PDF, also known as Version of record

Link back to DTU Orbit

Citation (APA):

Thamdrup, L. H., Persson, K. F., Bruus, H., Kristensen, A., \& Flyvbjerg, H. (2007). Experimental investigation of bubble formation during capillary filling of SiO2 nanoslits. Applied Physics Letters, 91(16), 163505.

https://doi.org/10.1063/1.2801397

\section{General rights}

Copyright and moral rights for the publications made accessible in the public portal are retained by the authors and/or other copyright owners and it is a condition of accessing publications that users recognise and abide by the legal requirements associated with these rights.

- Users may download and print one copy of any publication from the public portal for the purpose of private study or research.

- You may not further distribute the material or use it for any profit-making activity or commercial gain

- You may freely distribute the URL identifying the publication in the public portal 


\title{
Experimental investigation of bubble formation during capillary filling of $\mathrm{SiO}_{2}$ nanoslits
}

\author{
Lasse Højlund Thamdrup, Fredrik Persson, Henrik Bruus, and Anders Kristensen ${ }^{\text {a) }}$ \\ MIC-Department of Micro and Nanotechnology, Technical University of Denmark, Building 345 East, Ørsteds \\ Plads, DK-2800 Kongens Lyngby, Denmark \\ Henrik Flyvbjerg \\ Biosystems Department, Risф National Laboratory, Technical University of Denmark, Frederiksborgvej 399, \\ DK-4000 Roskilde, Denmark
}

(Received 15 August 2007; accepted 2 October 2007; published online 17 October 2007)

\begin{abstract}
Experimental results are presented regarding the influence of bubble formation on the capillary filling speed of water in $\mathrm{SiO}_{2}$ nanoslits with heights ranging from 33 to $158 \mathrm{~nm}$. The formation of an isolated pinned bubble in a nanoslit with a height of $111 \mathrm{~nm}$ causes an immediate decrease in the filling speed. In nanoslits with heights below $100 \mathrm{~nm}$, pinned bubbles are continuously formed at the advancing liquid meniscus. This observed increase in bubble density, which increases the fluidic resistance, quantitatively coincides with an observed reduction of the filling speed during filling of nanoslits with heights below $100 \mathrm{~nm}$. (C) 2007 American Institute of Physics.
\end{abstract}

[DOI: 10.1063/1.2801397]

Currently, passive nanofluidic structures are being adopted in order to investigate the statics and dynamics of confined double stranded DNA (dsDNA). Recent work include real-time contour length measurements on stretched dsDNA in nanochannels with cross sectional dimensions comparable to the biomolecule persistence length of $50 \mathrm{~nm},{ }^{1-3}$ entropically induced filtering of multidisperse dsDNA samples, and diffusivity measurements in nanoslit structures where only the height approaches the persistence length. ${ }^{5}$

Incorporating nanofluidic structures in lab-on-a-chip applications provides a powerful method of addressing dsDNA on a single molecule level, but also demands a thorough fundamental understanding of liquid dynamics in nanoconfinements. Capillary forces are prominent in nanofluidic structures due to the large surface-area-to-volume ratio. In Ref. 6 capillary filling measurements were conducted using microslits (the height $h \sim 1 \mu \mathrm{m}$ and the width $w>h$ ), and it was shown experimentally that the liquid dynamics could be described by the classical Washburn model modified to accommodate the rectangular cross section,

$$
L^{2}(t)=\left[\left(1-0.63 \frac{h}{w}\right) \frac{h \gamma \cos \theta}{3 \eta}\right] t \equiv a_{t} t .
$$

Here, $L$ is the propagated length of the meniscus and $\gamma, \eta$, and $\theta$ are the surface tension, viscosity, and advancing contact angle of the liquid, respectively. The term in the square brackets is the theoretically predicted constant of proportionality $a_{t}$.

It has been shown in Refs. 9 and 10 that capillary filling of fluidic structures with one or more dimensions of the order of $100 \mathrm{~nm}$ does not result in a qualitative deviation from the proportionality between the square of the propagation length and time proposed by the Washburn model. However, the liquid is observed to fill at a slower rate than expected. Thus, the measured constant of proportionality $a_{m}$ is smaller than $a_{t}$. The decrease in overall filling speed, which may be

\footnotetext{
${ }^{\text {a) }}$ Author to whom correspondence should be addressed. Electronic mail:
} ak@mic.dtu.dk. URL: www.optofluidics.dk, www.mic.dtu.dk/nil quantified by the ratio $a_{t} / a_{m}$, was in Ref. 10 interpreted as the result of electro-viscous retardation. However, bubble formation during capillary filling of nanoconfinements with polar solvents, such as de-ionized water, has frequently been reported, ${ }^{11-13}$ but the impact on the filling rate has not been experimentally addressed.

We have experimentally investigated the influence of bubble formation on the Washburn kinetics of de-ionized water $^{14}$ during capillary filling of 1 -cm-long, $10-\mu \mathrm{m}$-wide $\mathrm{SiO}_{2}$ nanoslits with heights ranging from 33 to $158 \mathrm{~nm}$ by recording the liquid progression $L$ as a function of time $t$. The nanoslits have been fabricated using a double thermal oxidation scheme previously presented in Ref. 15 . This technique makes it possible to fabricate devices with a nanoslit height-variation below $1 \mathrm{~nm}$ and a surface roughness of approximately $0.5 \mathrm{~nm}$ over an entire $4 \mathrm{in}$. wafer. A fusion bonding protocol ${ }^{15}$ was adopted in order to seal the fabricated devices using a Pyrex lid with sandblasted access holes for liquid introduction. A schematic of the device is shown in Fig. 1, along with an optical microscope image from a capillary filling experiment and a scanning electron microscope image of a nanoslit cross section. The bulk liquid reservoirs and ruler structures were defined using advanced silicon etching. ${ }^{16}$

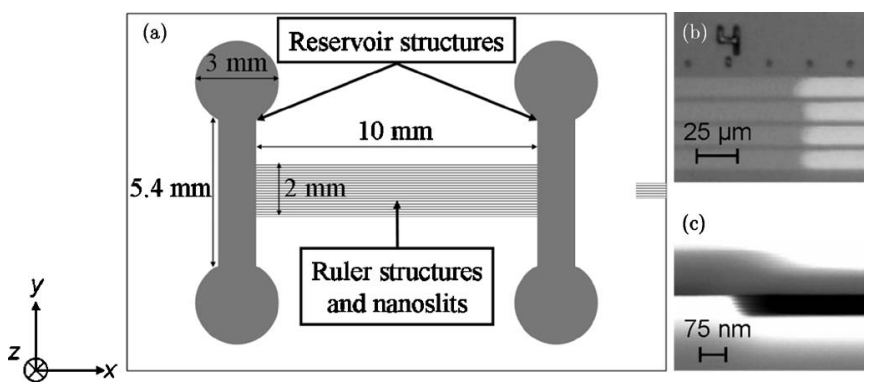

FIG. 1. (a) Schematic of the device used for the capillary filling experiments. The nanoslits are bridging the reservoir structures. (b) Microscope picture from a measurement on the capillary filling of nanoslits with $h$ $=111 \mathrm{~nm}$. By correlating the position of the liquid meniscus with tick marks on the ruler structures, the temporal progression of de-ionized water may be sampled. (c) Cross-sectional scanning electron microscope image of a sealed nanoslit with a height of $77 \mathrm{~nm}$. 
During the capillary filling, a constant wetting force $F_{w}$, provided by the Young-Laplace pressure drop across the curved meniscus, is counteracted by an increasing frictional contribution that is proportional to the propagated length $L$ and the filling speed $d L / d t$. In the absence of bubble formation, the capillary filling may therefore be modeled by the first order differential equation,

$$
F_{w}=\gamma_{0} L(t) \frac{d L(t)}{d t}
$$

where $\gamma_{0}$ is a constant that depends on the viscosity and the cross-sectional geometry. After imposing the proper boundary conditions, we obtain the analytical solution stated in the following equation from which it is seen that $a_{t}=2 F_{w} / \gamma_{0}$ and $L^{2} \propto t ;$

$$
L^{2}(t)=\frac{2 F_{w}}{\gamma_{0}} t
$$

as was the case in Eq. (1).

If a single, isolated pinned bubble is formed, an extra frictional contribution $\gamma_{b}$ must be added, since the bubble causes a local reduction of the nanoslit cross-sectional area which gives rise to an increase in the fluidic resistance. Hence, the wetting force can be written as

$$
F_{w}=\left[\gamma_{0} L(t)+\gamma_{b}(t)\right] \frac{d L(t)}{d t} .
$$

It may be assumed that $\gamma_{b}$ scales with the bubble volume $V_{b} \approx A_{b} h$, where $A_{b}$ is the area of the formed bubble projected into the $x y$ plane defined in Fig. 1. Furthermore, $V_{b}$ will decrease as a function of time as the local pressure $p$ at the position $L_{b}$, where the bubble is formed, increases as the liquid progresses in the nanoslit, i.e., $V_{b} \propto p^{-1}$, and, furthermore, the entrapped air will be dissolved in the liquid. Consequently, $\gamma_{b}$ is a decreasing function of time. In general, Eq. (4) may not be solved analytically since the exact functional expression of $\gamma_{b}(t)$ is unknown. However, it is evident from Eq. (4) that the formation of a bubble should cause a temporary decrease in the measured slope $a_{m, b}$ of the $L^{2}$ versus $t$ data. As the liquid progresses in the nanoslit after the bubble formation, $\gamma_{0} L(t)$ will dominate $\gamma_{b}(t)$ and $a_{m, b}$ will asymptotically approach the slope $a_{m, 0}$ measured before bubble formation. This effectively means that the temporal experimental signature of a single bubble may be revealed by analysis of the recorded $L^{2}$ versus $t$ data immediately before and after bubble formation.

Measurements were conducted on nanoslits with five different heights of 33, 48, 77, 111 and $158 \mathrm{~nm}$. During the filling of nanoslits with $h=158 \mathrm{~nm}$, no bubbles were formed. Careful analysis of the recorded movies during the filling of nanoslits with $h=111 \mathrm{~nm}$ revealed one preliminary case where exactly one pinned and isolated bubble is formed during the entire filling of a nanoslit. This allowed for evaluating the subtle experimental bubble signature. For nanoslits of $h=33,48$, and $77 \mathrm{~nm}$, bubbles were observed to form frequently due to air enclosure at the liquid meniscus. To ensure reproducibility, a strict protocol was adopted before initiating measurements. The filling experiments were conducted by dispersing a liquid droplet on an access hole and subsequently recording the meniscus progression $L$ in the nanoslits as a function of time $t$ using a charge-coupled device camera (Sony digital interface DFW-SX910) mounted on an upright Downloaded 18 Jun 2010 to 192.38.67.112. Redistribution subjec
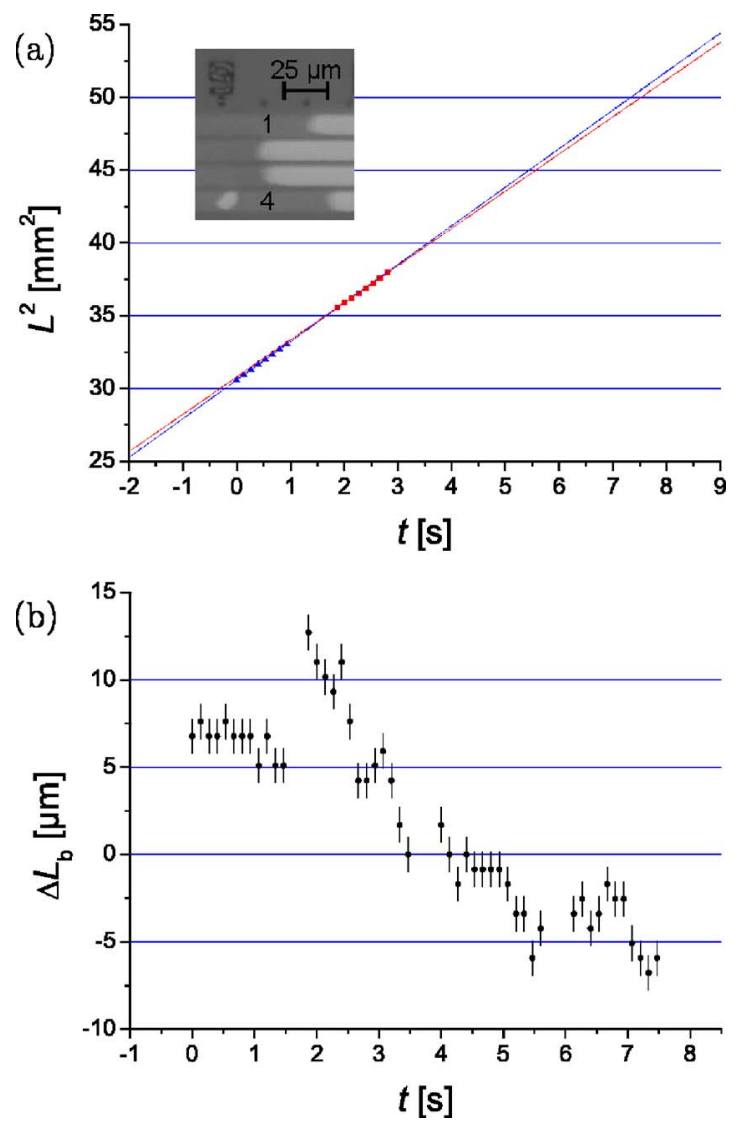

FIG. 2. (Color online) (a) The pinned bubble, shown in the micrograph insert, has an initial projected area $A_{b}=100 \mu \mathrm{m}^{2}$ and is formed during the capillary filling of a nanoslit with $w=10 \mu \mathrm{m}$ and $h=111 \mathrm{~nm}$ at $L_{b}$ $=5.9 \mathrm{~mm}$. The triangles and squares in the $L^{2}$ vs $t$ graph indicate measurements immediately before and after the bubble is formed, respectively. Notice the decrease in slope. Before bubble formation $a_{m, 0}=2.65 \mathrm{~mm}^{2} / \mathrm{s}$ and after $a_{m, \mathrm{~b}}=2.56 \mathrm{~mm}^{2} / \mathrm{s}$. (b) The distance $\Delta L_{\mathrm{b}}$ between the meniscus position in nanoslit 4 (with a bubble) and nanoslit 1 (without a bubble) as a function of time. Notice the forward jump of approximately $7 \mu \mathrm{m}$ immediately after bubble formation and the converging value of $\Delta L_{b}=-5 \mu \mathrm{m}$.

microscope fitted with a Leica ApoZoom 1:6 objective. In analyzing the experimental signature of the bubble formed during capillary filling of the nanoslit with $h=111 \mathrm{~nm}$, the slopes of linear regressions to eight consecutive data points immediately before and after bubble formation (i.e., $a_{m, 0}$ and $a_{m, b}$, respectively) were determined. Furthermore, the difference in propagated length $\Delta L_{b}$ between the nanoslit in which the bubble was formed and a reference nanoslit was monitored at 12 consecutive values of $t$ immediately before bubble formation and 37 values of $t$ immediately after. The overall values of $a_{m}$ of the $L^{2}$ versus $t$ data for all nanoslit heights were evaluated based on the average slope of a linear regression to eleven data points in the interval $2 \mathrm{~mm}<L$ $<8.5 \mathrm{~mm}$ in order to determine the ratio $a_{t} / a_{m}$ central to this study. For all heights the average slope was based on analysis of filling data from 24 individual nanoslits.

Initially we present results regarding the experimental signature of the formation of an isolated pinned bubble in the $L^{2}$ versus $t$ data obtained during capillary filling measurements in a nanoslit with $h=111 \mathrm{~nm}$. The bubble has an initial projected area $A_{b} \sim 100 \mu \mathrm{m}^{2}$. Sampling the meniscus position inside the nanoslit in a continuous image sequence immediately before and after the bubble is formed allows for determining the impact on the measured slopes $a_{m, 0}$ and $a_{m, b}$ by performing linear regressions to the $L^{2}$ versus $t$ data, as to AlP license or copyright; see http://apl.aip.org/apl/copyright.jsp 


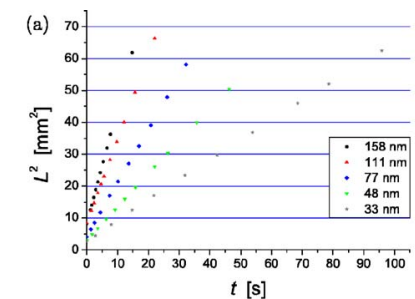

(c)

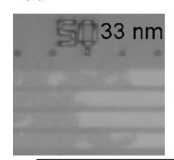

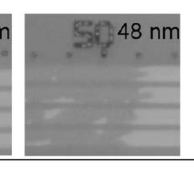

$h[\mathrm{~nm}]$

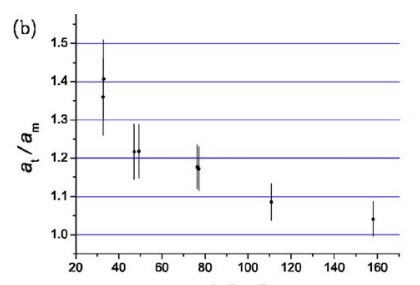

F. 3. (Color online) (a) The raw filling data $L^{2}$ vs $t$ sampled from randomly selected nanoslits with $h=33-158 \mathrm{~nm}$. No overall deviation from $L^{2} \propto t$ was observed. (b) The ratio $a_{t} / a_{m}$ as a function of nanoslit height $h$. Notice how the ratio increases substantially for $h<100 \mathrm{~nm}$. (c) Still images taken at $L \sim 5.0 \mathrm{~mm}$ during capillary filling experiments. Notice how lowering the nanoslit height causes a more distorted meniscus shape in the width direction and, furthermore, leads to an increase in bubble density.

seen in Fig. 2(a). Moreover, the difference $\Delta L_{b}$ of the meniscus position in a nanoslit with a bubble and one without is monitored as a function of time [see Fig. 2(b)]. The experimental signature of the presence of a bubble has three distinct characteristics: (i) The measured slope decreases temporarily by $4 \%$ [see Fig. 2(a)], (ii) an abrupt forward-jump $\Delta L_{\mathrm{fj}}$ of approximately $7 \mu \mathrm{m}$ in the position of the meniscus is evident [see Fig. 2(b)], and (iii) approximately $5 \mathrm{~s}$ after the bubble formation the liquid progresses at the same speed as the liquid in the reference nanoslit. The latter is indicated by a converging value of $\Delta L_{b} \approx-5 \mu \mathrm{m}$ seen in Fig. 2(b). The decrease in slope is due to a local increase in friction induced by the formed bubble as it narrows the nanoslit, whereas the size of the forward jump of the meniscus is approximately related to the initial size of the bubble as $A_{b} \approx \Delta L_{\mathrm{fj}} w$. The effect of the bubble formation is local in time and is gone after approximately $5 \mathrm{~s}$. However, the net impact of the formation of a single isolated bubble will be a minute increase in the overall nanoslit filling time.

Next, the correlation between observed bubble density and the experimentally determined ratio $a_{t} / a_{m}$ is considered. From the graph of $a_{t} / a_{m}$ as a function of nanoslit height $h$ shown in Fig. 3(b), it is evident that for $h<100 \mathrm{~nm}$ a large and systematic increase in the ratio is observed. Furthermore, it should be noted that $L^{2}$ and $t$ remain proportional [refer to Fig. 3(a)] also when filling occurs at a slower rate, as indicated by the nonunity values of $a_{t} / a_{m}$. Both of these empirical observations support and reproduce the work presented in. Refs. 10 and 15. During filling experiments of nanoslits with $h>100 \mathrm{~nm}$, bubble formation occurred rarely and the meniscus shape in the $y$ direction (refer to coordinate system in Fig. 1) was undistorted except for minor fluctuations. As evident from the image sequence in Fig. 3(c), decreasing $h$ below $100 \mathrm{~nm}$ results in a distorted meniscus shape and, furthermore, a quantitative increase in pinned bubble density. Decreasing the height has the effect of increasing the YoungLaplace pressure drop and lowering the mean velocity of the propagating liquid. This is observed to promote the formation of pinned bubbles. It is far from being trivial to evaluate how this increased bubble density influences the overall filling rate since this would call for a model implementing the stochastic bubble formation and also possible synergetic or collaborative effects between multiple small scale bubbles.
However, based on the findings regarding the experimental significance of a single bubble, it is plausible that the increased density of pinned bubbles will lead to an overall increase in the fluidic resistance of the nanoslit and this ultimately results in a reduced filling rate. It is important to distinguish this effect from an increase in effective/apparent viscosity of the liquid caused either by dynamic bubbles moving along with the propagating liquid or by other effects, e.g., electroviscous retardation. The increase in fluidic resistance, caused by small scale pinned bubbles, might provide an alternative or complementary explanation to the increase in $a_{t} / a_{m}$, observed experimentally for nanoslits with $h<100 \mathrm{~nm}$, an increase which has primarily been interpreted as the result of electroviscous retardation. ${ }^{10}$

In conclusion it has been shown experimentally that the formation of an isolated pinned bubble in a nanoslit with $h=111 \mathrm{~nm}$ causes a short-time reduction of the filling speed and is accompanied by a forward jump of the meniscus. We have also established a quantitative correlation between the increased bubble density observed in nanoslits with $h<100 \mathrm{~nm}$ and the decrease in the significant ratio $a_{t} / a_{m}$. Considering the impact of a single bubble on the liquid dynamics, the increased bubble density will result in an overall increase in fluidic resistance. In our experiments the filling rate has been high compared to the diffusion coefficient of air in water. However, one should be aware of the fact that as $h$ is lowered into the sub- $10 \mathrm{~nm}$ regime, diffusion of entrapped air into the liquid could result in an overall reduction of the bulk viscosity. Clearly this would effectively serve as to increase the filling rate.

This work was supported by the Danish Research Council for Technology and Production (FTP Grant No: 274050375). The authors acknowledge Jakob G. Howalt and Jakob B. Larsen for experimental assistance.

${ }^{1}$ W. Reisner, K. Morton, R. Riehn, Y. Wang, Z. Yu, M. Rosen, J. Sturm, S. Chou, E. Frey, and R. Austin, Phys. Rev. Lett. 94, 196101 (2005).

${ }^{2}$ J. Tegenfeldt, C. Prinz, and H. Cao, Proc. Natl. Acad. Sci. U.S.A. 101, 10979 (2004).

${ }^{3}$ W. Reisner, J. Beech, N. Larsen, H. Flyvbjerg, A. Kristensen, and J. Tegenfeldt, Phys. Rev. Lett. 99, 058302 (2007).

${ }^{4}$ J. Fu, J. Yoo, and J. Han, Phys. Rev. Lett. 97, 018103 (2006).

${ }^{5}$ A. Balducci, P. Mao, J. Han, and P. Doyle, Macromolecules 39, 6273 (2006).

${ }^{6}$ L. Yang, T. Yao, and Y. Tai, J. Micromech. Microeng. 14, 220 (2004).

${ }^{7}$ E. Washburn, Phys. Rev. 17, 273 (1921).

${ }^{8} \mathrm{H}$. Bruus, Theoretical Microfluidics 1st ed. (Oxford University Press, Oxford, 2007).

${ }^{9}$ A. Hibara, T. Saito, H. Kim, M. Tokeshi, T. Ooi, M. Nakao, and T. Kitamori, Anal. Chem. 74, 6170 (2002).

${ }^{10}$ N. Tas, J. Haneveld, H. Jansen, M. Elwenspoek, and A. van den Berg, Appl. Phys. Lett. 85, 3274 (2004).

${ }^{11}$ J. Eijkel, J. Bomer, N. Tas, and A. van den Berg, Lab Chip 4, 161 (2004).

${ }^{12}$ A. Han, G. Mondin, N. Hegelbach, N. de Rooij, and U. Staufer, J. Colloid Interface Sci. 293, 151 (2006).

${ }^{13}$ M. Rossi, H. Ye, Y. Gogotsi, S. Babu, P. Ndungu, and J. Bradley, Nano Lett. 4, 989 (2004).

${ }^{14}$ The Milli-Q water was from a dedicated MILLIPORE Milli-Q gradient A10 system with conductivity $\sigma_{\mathrm{el}}=5.5 \times 10^{-6} \pm 0.3 \mathrm{~S} / \mathrm{m}$, surface tension $\gamma=73 \pm 2 \mathrm{~mJ} / \mathrm{m}^{2}$, and viscosity $\eta=0.89 \pm 0.02 \mathrm{mPa} / \mathrm{s}$. We use that $\cos \theta=\frac{1}{2}\left(\cos \theta_{\mathrm{SiO}_{2}}+\cos \theta_{\text {Pyrex }}\right)=0.94 \pm 0.02$. All measurements were carried out within $2-5 \mathrm{~h}$ after the contact angle measurements at a temperature of $22-24{ }^{\circ} \mathrm{C}$.

${ }^{15}$ F. Persson, L. Thamdrup, M. Mikkelsen, S. Jarlgaard, P. Skafte-Pedersen, H. Bruus, and A. Kristensen, Nanotechnology 18, 245301 (2007).

${ }^{16}$ Trademark of Surface Technology Systems Limited (STS), UK (www.stsystems.com). 